\title{
BRITISH PLANS FOR ASTRONOMY IN ANTARCTICA
}

\author{
R D Davies, University of Manchester \\ J M Hough, Hatfield Polytechnic
}

\section{Present Activity.}

The South Pole air shower experiment (SPASE), a joint Bartol Research Institute and Leeds University project, has been operational since the austral summer of 1987/88. It is a cosmic ray telescope searching for cosmic gamma rays at energies up to $1000 \mathrm{TeV}$. Although it has a relatively small area $\left(6800 \mathrm{~m}^{2}\right)$, it is situated at an altitude of $2800 \mathrm{~m}$ and has a 24 hour coverage, making it very competitive. The angular resolution of 0.8 at $200 \mathrm{TeV}$ is state-ofthe-art in gamma ray astronomy. The astronomical programme includes searches for gamma ray sources, searches for anisotropy in the cosmic ray sky and measuring the energy spectrum over the range $10^{14}-10^{16} \mathrm{eV}$.

\section{Planned Activity.}

During the 1991/92 austral summer, a large area muon detector will be constructed consisting of 8 holes in the ice sheet each $200 \mathrm{~m}$ deep, instrumented with photomultiplier Cerenkov detectors. This development is a collaboration between Bartol/Leeds and groups from the University of Wisconsin (Madison) and California (Irvine). Further additions will be made to the SPASE array.

Another initiative in the next two years is the expected move of the Jodrell Bank 32GHz CMB anisotropy experiment from Tenerife to the British Antarctic Survey site at Halley Base. It is hoped to overwinter this equipment to provide a sufficiently long observing period to obtain a sensitivity of $\Delta \mathrm{T} / \mathrm{T}=2-3 \times 10^{-6}$ over half a steradian of sky with a resolution of $5^{\circ}$. This experiment could benefit from a longer period of observations at a South Pole site with lower water vapour content than Halley Base.

\section{Possible Future Developments.}

A team from Queen Mary and Westfield, Jodrell Bank, MRAO Cambridge and Lancashire Polytechnic are actively planning CMB anisotropy experiments on $1^{\circ}$ and $5^{\circ}$ scales at 1,2 and $3 \mathrm{~mm}$ wavelengths. A prototype bolometer system cooled to helium 3 temperatures is to be tested on Mauna Kea during the coming winter. This system would take advantage of the very low humidity of a South Pole site. Another development being considered which would greatly benefit from such a site is an MRAO/Jodrell Bank proposal for a millimetre array for CMB anisotropy studies from a few arc minutes to one degree.

The Birmingham University solar/stellar seismology group has interests in a polar station which provides superior spectroscopy and photometry over long periods. Their astronomical objectives include (a) stellar seismology of solar-type and main sequence stars (b) search for planetary systems orbiting main sequence stars - of heightened interest following the Jodrell Bank discovery of a planet outside the solar system and (c) a further investigation of the solar acoustic continuum simultaneously with other stations.

Further UK interests include sub-millimetre astronomy, a long baseline millimetre array and near infrared astronomy, all of which may benefit from the dry Antarctic conditions. 